\title{
THE RELATIONSHIP OF THE Oe TO THE Be STARS
}

\author{
STEWART A. FROST* and PETER S. CONTI** \\ Joint Institute for Laboratory Astrophysics \\ University of Colorado and National Bureau of Standards \\ and \\ Dept. of Physics and Astrophysics \\ University of Colorado, Boulder, Color., U.S.A.
}

\begin{abstract}
Oe stars are earlier type analogues of the better known Be class. These stars have relatively narrow emission in the hydrogen lines and sometimes in the helium lines, which often appears to be double. In several Oe stars, the emission is intermittent. Other properties of the class include relatively broad absorption lines and luminosities near the main sequence. Line profiles in three representative $\mathrm{Oe}$ stars are presented and discussed. The similarity of the spectra of Oe and $\mathrm{Be}$ stars suggests that the formation mechanism for the emitting region, whatever it is, must extend to the mid O-type stars, at least.
\end{abstract}

Recently, Conti and Leep (1974) introduced the spectral classification type Oe for those O-type stars showing emission due to the Balmer lines of hydrogen and not showing emission due to $\mathrm{N}_{\text {III }} \lambda \lambda 4634,40,41 \AA$ and $\mathrm{He}$ II $\lambda 4686 \AA$. The spectral types of the stars that they included in this class are given in Table I. One additional

TABLE I

Spectral types of the Oe stars

\begin{tabular}{|c|c|c|c|c|}
\hline & MWC & Conti and Leep & Walborn & Morgan et al. \\
\hline $\begin{array}{l}\text { X Per } \\
\text { HD 39680 } \\
\text { HD } 45314 \\
\text { HD } 46056 \\
\text { HD } 60848 \\
\zeta \text { Oph } \\
\text { HD 155806 } \\
68 \text { Cyg }\end{array}$ & $\begin{array}{r}78 \\
783 \\
140 \\
808 \\
184 \\
\\
\\
252\end{array}$ & $\begin{array}{l}\text { OBe } \\
\text { OBe } \\
\text { O8 V(e) } \\
\text { O9 V(e) } \\
\text { O7.5 IIIe } \\
\text { O8 V }\end{array}$ & $\begin{array}{l}\text { O6 V: [n]pe var } \\
\text { O8 Vn } \\
\text { O7.5 V[n]pe } \\
\text { O7.5 III : n((f)) }\end{array}$ & O8 V : pe \\
\hline
\end{tabular}

star, $68 \mathrm{Cyg}$, has been included for reasons which will be mentioned later. The spectral types are taken from Walborn $(1972,1973)$ and Conti and Leep (1974), except for that of HD 60848 which is taken from Morgan et al. (1955). It is important to note that all of these stars have spectra which indicate that they are on or near the main sequence. The appearance of the line profiles of $\mathrm{He}_{\text {II }} \lambda 4686 \AA$, some of which will be illustrated later, are consistent with these classifications. In addition, two of these stars, HD 46056 and $\zeta$ Oph, have well established absolute magnitudes, and these magnitudes indicate that they are on the main sequence. The Mount Wilson Catalogue numbers (Merrill and Burwell, 1949) are also listed in Table I. All of these

* Also Visiting Student, Kitt Peak National Observatory, which is operated by AURA, Inc., under contract with the National Science Foundation.

** Also Department of Astrogeophysics, University of Colorado; Guest Investigator, Hale Observatories; and Visiting Astronomer, Kitt Peak National Observatory, which is operated by AURA, Inc., under contract with the National Science Foundation. 
stars, except $\zeta$ Oph and 68 Cyg were observed to have hydrogen Balmer line emission more than twenty-five years ago. However, Merrill and Burwell noted that the emission in HD 46056 was present intermittently. We will present line profiles for HD 39680, HD 60848, and HD 155806.

The spectrograms from which the line profiles were obtained were taken during the years 1972-1974 at the Kitt Peak National Observatory, Palomar Mountain Observatory, and Mount Wilson Observatory. The coudé spectrographs were employed so as to give a dispersion of about $18 \AA \mathrm{mm}^{-1}$, on nitrogen baked IIa-O, in the $\lambda 3700-4900 \AA$, blue region, and a dispersion of $25 \AA \mathrm{mm}^{-1}$, on unbaked and nitrogen baked 098-04, in the $\lambda$ 5000-6800 $\AA$, yellow-red region. The smoothed line profiles were produced from the spectrograms by the computer method described by Conti and Frost (1974). The spectra were traced on a microphotometer. The digital density information was filtered by a fast Fourier transform method (Brault and White, 1971) and converted to intensity through the use of a polynomial calibration curve. The line profiles were calculated and plotted on microfilm by computer, and later copied by hand from prints of the film. The zero point of the velocity scale was determined for each spectrum from a number of absorption lines. The line profiles were sometimes shifted $50-100 \mathrm{~km} \mathrm{~s}^{-1}$ in order to obtain the 'best' agreement between a number of them.

The Balmer line profiles, shown in Figures 1-4, are similar to the double peaked or central emission line profiles characteristic of the Be stars. The emission is strongest at $\mathrm{H} \alpha$ and decreases in strength as one goes to the shorter wavelength lines. For the

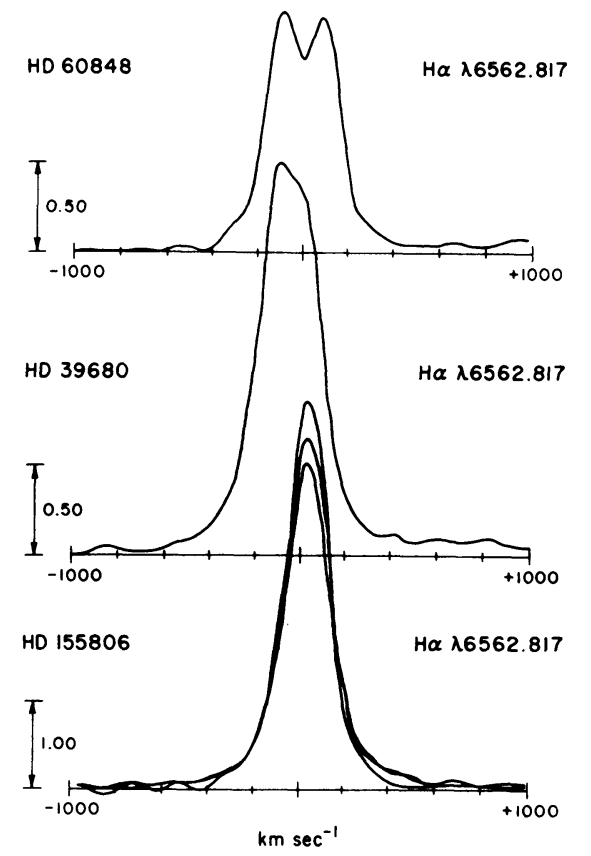

Fig. 1. Normalized, filtered $\mathrm{H} \alpha$ line profiles in three Oe stars. In this figure, and all subsequent ones, the zero point of the velocity scale is only approximately correct. Shifts in line center from $0 \mathrm{~km} \mathrm{~s}^{-1}$ may not be real. 


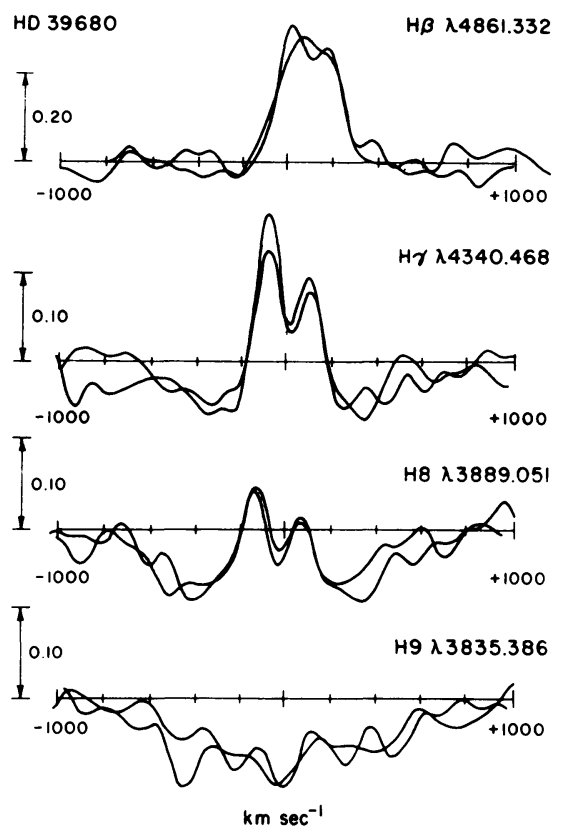

Fig. 2. Normalized, filtered Balmer line profiles in HD 39680. The emission has disappeared at $\mathrm{H} 9$ and only the underlying photospheric feature remains.

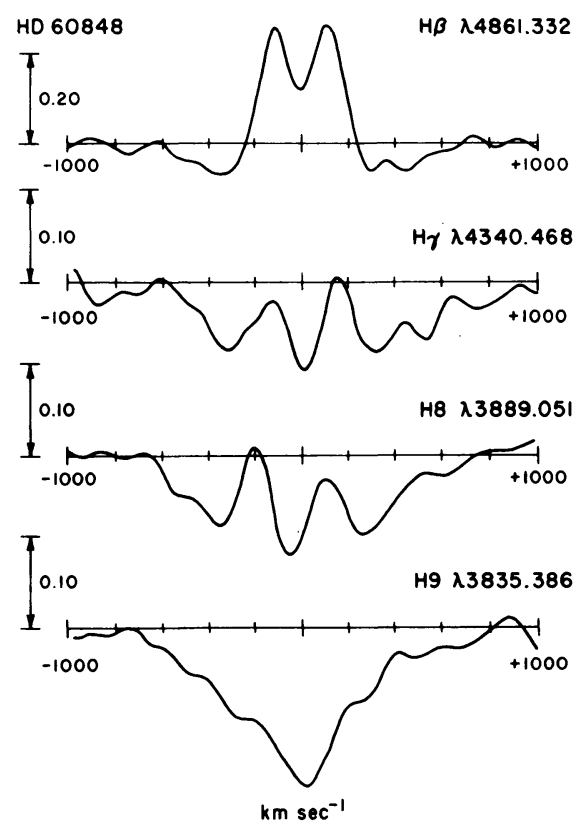

Fig. 3. Normalized, filtered Balmer line profiles in HD 60848. The emission has disappeared at $\mathrm{H} 9$ and only the underlying photospheric feature remains. 


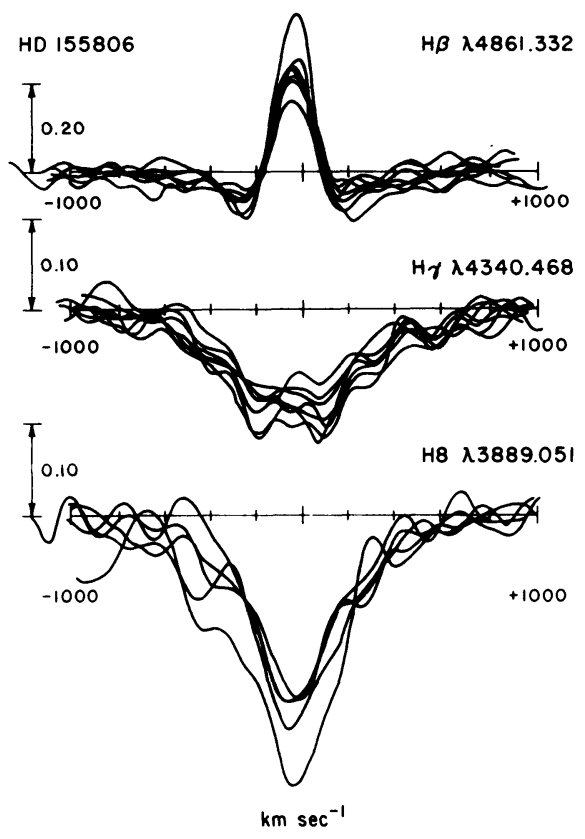

Fig. 4. Normalized, filtered Balmer line profiles in HD 155806. The emission has essentially disappeared by $\mathrm{H} \gamma$ and only the underlying photospheric feature remains. Note, however, that $\mathrm{H} \alpha$ was much stronger in this star than in the other two as shown by Figure 1.

higher members of the Balmer series, $\mathrm{H} \gamma$ and $\mathrm{H} 8$ in $\mathrm{HD} 39680, \mathrm{H} \beta, \mathrm{H} \gamma$, and $\mathrm{H} 8$ in HD 60848 , and $H \beta$ in 155086 , one clearly sees the profiles as the superposition of emission on a broader absorption. During the period of time covered by these observations, the Balmer line profiles appear not to have varied very much.

Figures 5-7 illustrate the triplet and singlet $2 P^{0}-n D$ series of neutral helium. The velocity scales for $\lambda 5876 \AA$ and $\lambda 6678 \AA$ are compressed by a factor of two relative to the velocity scales for the other lines. In HD 39680 and HD 60848, $\lambda 5876 \AA$, the first member of the triplet series, and $\lambda 6678 \AA$, the first member of the singlet series, appear in emission. Similar emission was noted by Hutchings (1970) in $\gamma$ Cas and Bahng and Hendry (1975) in $\kappa \mathrm{CMa}$, both Be stars. The appearance of the triplet line, $\mathrm{He}_{\mathrm{I}} \lambda 4471 \AA$, is of interest in both of these stars. Relative to the next triplet line, $\lambda 4026 \AA$, it appears to be partially filled in by emission. Thus, in addition to the first members of the triplet and singlet series, $\mathrm{He}_{\mathrm{I}} \lambda 4471 \AA \AA$ seems to be formed in an extended atmosphere. Unfortunately, observations of the singlet line, He I $\lambda 4921 \AA$ are not yet available. The higher series members appear as broad absorption lines. The behavior of the $2 P^{0}-n D$ series of neutral helium in these two stars is in some ways similar to that of the Balmer series of hydrogen. In HD 155806, however, all the $\mathrm{He} \mathrm{I}$ lines are in absorption.

Spectral classification employing the helium ionization balance as given by the ratio of the equivalent widths of $\mathrm{He}_{\text {I }} \lambda 4471 \AA$ to $\mathrm{He}_{\text {II }} \lambda 4541 \AA$ will give spectral types which are too early for some Oe stars. In HD 39680, the He II lines indicate a spectral type near $\mathrm{O} 9$, in contrast to the earlier spectral type found by Walborn 

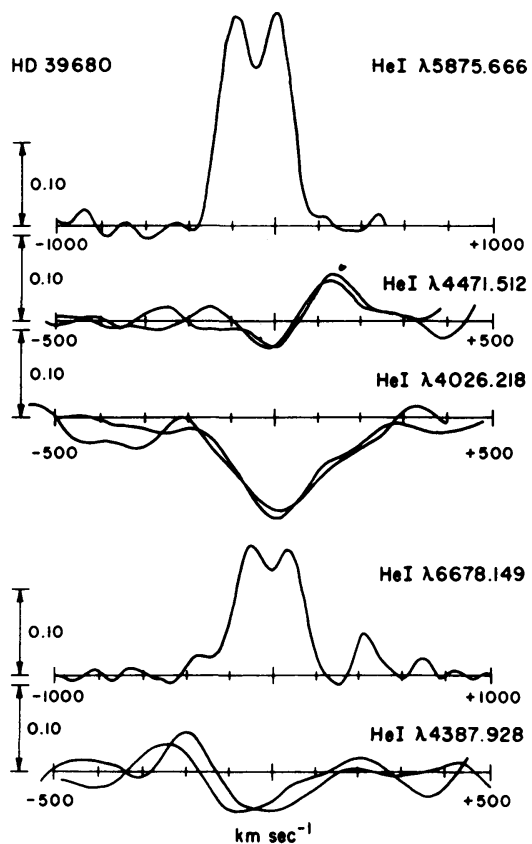

Fig. 5. Normalized, filtered neutral helium lines in HD 39680. The emission has disappeared at the third member of the series.


Fig. 6. Normalized, filtered neutral helium lines in HD 60848. The emission has disappeared at the third member of the series. 


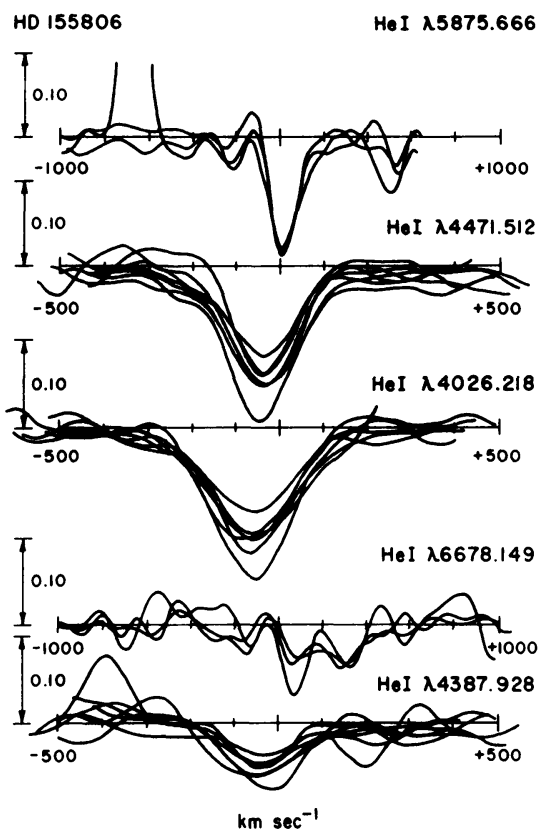

Fig. 7. Normalized, filtered neutral helium lines in HD 155806. No emission is present.

(1973). Thus, HD 155806, with a spectral type of O7.5, is the earliest Oe star of which we are aware.

These three stars are O-type stars, as seen by the presence of the lines due to singly ionized helium shown in Figures 8-10. All of these features appear as broad absorption lines. It is important to note that He II $\lambda 4686 \AA$ appears strongly in absorption, implying that it is formed in a plane parallel region. In addition, the $\mathrm{N}$ III complex is not present in emission in these stars. This is in contrast to the behavior of these lines in the Of stars.

Thus, these three Oe stars appear to be spectroscopically similar to the Be stars. Their spectra contain emission lines, due to hydrogen and neutral helium, arising in a rotating envelope and rotationally broadened absorption lines.

Massa (1975) considered the interesting question of the significance of radiation pressure in the appearance of the Be phenomenon. He concluded that the presence of this force aids in the formation of a disk. That is, as the magnitude of the force increases, presumably as one goes to earlier spectral types, the amount of rotation necessary for the formation of a circumstellar envelope decreases. Massa found that roughly $20 \%$ of the B1-5 stars and $12 \%$ of the stars earlier than B1 have shown emission at some time. He felt that there is a smaller fraction of Be stars at the earlier spectral types, but did state that this may be an observational effect due to the intermittent nature of the emission in the hotter stars. He suggested that at the earliest spectral types the relationship between the magnitude of the radiation pressure and the appearance of Be type emission may have broken down. 


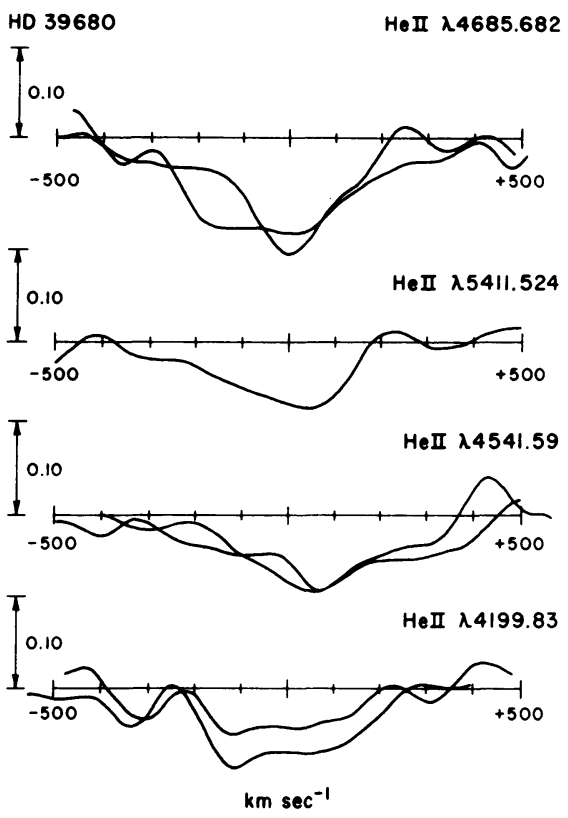

Fig. 8. Normalized, filtered ionized helium lines in HD 39680. No emission is present.



Fig. 9. Normalized, filtered ionized helium lines in HD 60848. No emission is present. 


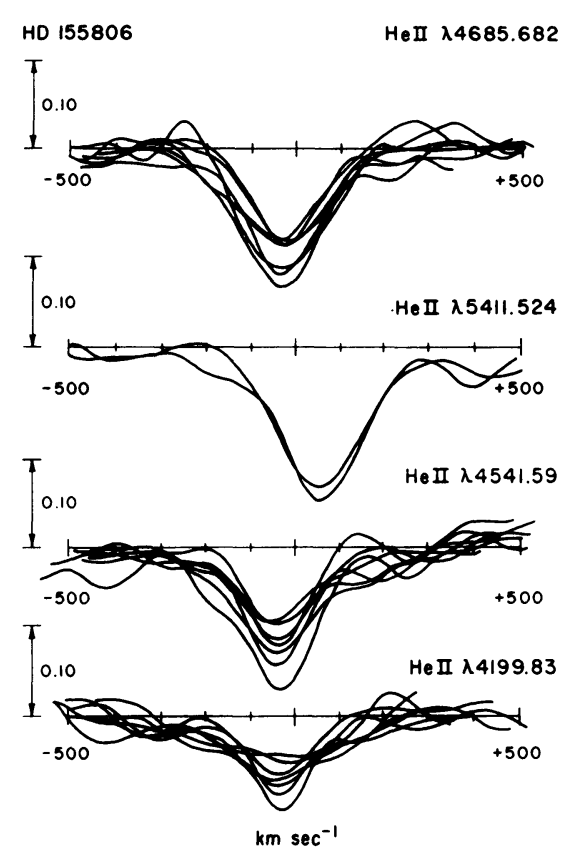

Fig. 10. Normalized, filtered ionized helium lines in HD 155806. No emission is present.

There are 28 stars in the O star survey of Conti and Alschuler (1971) which meet the following requirements: (1) later than O7.5 (2) on the main sequence (3) $m_{v} \leq 8.0$ and (4) $\delta>-20^{\circ}$. Of these, two, X Per and HD 45314, are Oe stars. Two others, $\zeta$ Oph (Niemelä and Méndez, 1974; Barker and Brown, 1974) and 68 Cyg (Wilson, 1958) have shown intermittent emission only at $\mathrm{H} \alpha$, and are classified $\mathrm{O}(\mathrm{e})$ according to the spectral types of Conti and Leep (1974). For the O-type stars of this statistically small sample, $14 \%$ have exhibited the Oe phenomenon at some time. That this fraction is similar to that for the B-type stars is probably not significant considering the paucity of the statistics. One wonders whether the Oe stars have large winds and how a circumstellar disk would be maintained in the presence of such a wind.

Some of the Oe stars exhibit hydrogen Balmer line emission only at irregular intervals. This activity is very similar to that observed in some Be stars. The behavior of the Oe and Be stars is not like that of the Of stars in this regard. That is, there are no O-type stars known to us which have shown Of type emission intermittently. This indicates that the physical mechanisms underlying the Oe and Of phenomena are very different indeed.

\section{Acknowledgments}

This research has been supported by the NSF under grant No. MPS72-05062 A02. Acknowledgment is also made to the National Center for Atmospheric Research, which is sponsored by the National Science Foundation, for computer time used in this research. 


\section{References}

Bahng, J. D. R. and Hendry, E.: 1975, Publ. Astron. Soc. Pacific 87, 137.

Barker, P. K. and Brown, T.: 1974, Astrophys. J. Letters 192, L11.

Brault, J. W. and White, O. R.: 1971, Astron. Astrophys. 13, 169.

Conti, P. S. and Alschuler, W. R.: 1971, Astrophys. J. 170, 325.

Conti, P. S. and Frost, S. A.: 1974, Astrophys. J. Letters 190, L137.

Conti, P. S. and Leep, E. M.: 1974, Astrophys. J. 193, 113.

Hutchings, J. B.: 1970, Monthly Notices Roy. Astron. Soc. 150, 55.

Massa, D.: 1975, Publ. Astron. Soc. Pacific 87, 777.

Merrill, P. W. and Burwell, C. G.: 1949, Astrophys. J. 110, 387.

Morgan, W. W., Code, A. D., and Whitford, A. E.: 1955, Astrophys. J. Suppl. Ser. 2, 41.

Niemelä, V. S. and Méndez, R. H.: 1974, Astrophys. J. Letters 187, L23.

Walborn, N.: 1972, Astron. J. 77, 312.

Walborn, N.: 1973, Astron. J. 78, 1067.

Wilson, R.: 1958, Publ. Roy. Obs. Edinburgh 2, 61.

\section{DISCUSSION}

Hutchings: Are there variable radial velocities for any of these stars?

Conti: There is very little radial velocity information in the catalogues on these Oe stars. However, both $\zeta$ Oph and $68 \mathrm{Cyg}$ do seem to have non-negligible ranges in radial velocity.

Hutchings: I suggest you look at the Balmer decrements and peak separation progressions in Oe stars to see if X Per is typical of Oe types or still different from all the rest. 